\title{
A retrosigmoid ileal conduit might prevent ureteroileal anastomotic stricture after ileal conduit diversion
}

\author{
Cristiano Mendes Gomes, Luccas Soares Laferreira \\ Division of Urology, Department of Surgery, University of Sao Paulo School of Medicine, Sao Paulo, Brasil \\ Correspondence to: Cristiano Mendes Gomes. Division of Urology, Hospital das Clinicas da Faculdade de Medicina da Universidade de Sao Paulo, \\ Avenida Dr. Enéas de Carvalho Aguiar, 255, Sala 710F-7º, Andar, CEP 05403-000, São Paulo, SP, Brasil. Email: crismgomes@uol.com.br. \\ Provenance: This is an Invited Editorial commissioned by Section Editor Xiao Li (Department of Urology, Jiangsu Cancer Hospital \& Jiangsu \\ Institute of Cancer Research \& Nanjing Medical University Affiliated Cancer Hospital, Nanjing, China). \\ Comment on: Ficarra V, Giannarini G, Crestani A, et al. Retrosigmoid Versus Traditional Ileal Conduit for Urinary Diversion After Radical \\ Cystectomy. Eur Urol 2018. [Epub ahead of print].
}

Submitted Oct 09, 2018. Accepted for publication Oct 12, 2018.

doi: $10.21037 /$ tau.2018.10.10

View this article at: http://dx.doi.org/10.21037/tau.2018.10.10

\section{Introduction}

Ureteroenteric anastomotic (UEA) stricture remains a significant complication after continent or incontinent urinary diversion. Most commonly, the left ureteral implantation is affected, which is thought to be caused by a wider mobilization of the ureter (1). The retrosigmoid tunnel may also increase the risk for angulation and/or compression of the left ureter. In this study (2), the authors presented their results with a technique involving the retrosigmoid transposition of the ileal conduit from the right to the left side of the body in order to avoid the problems associated with the crossing of the left ureter to the right side.

\section{Ureteroileal strictures after ileal conduit diversion: incidence and management}

UEA stricture rates range from $2.6 \%$ to $14 \%(3-6)$ with a median postoperative time of 6 to 18 months (4-6). Studies have shown that the left ureter is involved in up to $80 \%$ of the cases $(6,7)$. UEA strictures are likely to be caused by ischemia and inflammation, which may be greater in the left side due to excessive mobilization and tension on the ureter caused by the transposition of the left ureter through the sigmoid mesocolon, resulting in fibrosis and ureteral obstruction. Meticulous surgical technique, preservation of periureteral adventitia and blood supply, avoidance of electrocautery, and excision of the compromised distal ureter before anastomosis are common strategies employed to lessen the risks of UEA stricture (8).

Management of UEA strictures is challenging, with several options described for the initial surgical approach. The traditional treatment for ureteroileal stricture is open surgical repair, which is usually a difficult procedure because the anatomy is often distorted with abundant scarring. Although very good success rates of up to $90 \%$ have been reported (5), serious complications (Clavien $\geq 3 \mathrm{~b}$ ) occur in $7-13 \%$ including significant bleeding requiring transfusion, vascular or intestinal perforation and high reoperation rates (5,7,9-11).

Endourological techniques are often performed as firstline treatment as less invasive alternatives to open surgical revision of UEA strictures. However, they have been scarcely investigated and the results seem to be suboptimal. A recent study found $23 \%$ success rate for endourological techniques and $87 \%$ success rate for open surgical revision anastomosis at a median follow-up of 33 months (12). These results are in line with previous studies reporting success rates of $0-50 \%$ with endourological techniques $(5,7,10)$ and $76-93 \%$ with open surgical revision $(4,7,11,13)$. Although the complication rates are certainly lower with the endourological approach, life-threatening complications have been reported (14).

\section{Potential advantages of the retrosigmoid ileal conduit and comparison with other techniques}

Ureteroileal strictures are more common in the left ureter 
Table 1 Comparing the results of different techniques in terms of ureteroileal strictures

\begin{tabular}{|c|c|c|c|c|c|}
\hline References & $\mathrm{n}$ & Technique & Mean follow-up (months) & Total strictures (\%) & Left ureter $(\%)$ \\
\hline Evangelidis et al., 2006 (19) & 198 & Traditional ileal conduit & 19.9 & $8(4.0)$ & $7(63.6)^{\star}$ \\
\hline Liu et al., 2014 (20) & 99 & Traditional ileal conduit & 30.2 & $6(6.1)$ & $4(66.7)$ \\
\hline Desai et al., 2014 (21) & 132 & Traditional ileal conduit & 3 & $5(3.7)$ & Not reported \\
\hline Pagano et al., 2005 (18) & 100 & Anterior ileal conduit & 57.6 & $5(5)$ & Not reported \\
\hline Li et al., 2011 (16) & 42 & Retrosigmoid ileal conduit & 18.6 & 0 & 0 \\
\hline Kotb et al., 2013 (15) & 40 & Retrosigmoid ileal conduit & 12 & 0 & 0 \\
\hline Ficarra et al., 2018 (2) & 30 & Retrosigmoid ileal conduit & 10.8 & 0 & 0 \\
\hline
\end{tabular}

*, 3 patients with bilateral strictures, totalizing 11 ureteral units.

and the wider mobilization required to transpose it to the right side through a retrosigmoid tunnel is supposed to lead to ischemia and to increase the risk of stricture. In addition, the retrosigmoid tunnel may also increase the risk for angulation and/or compression of the left ureter. Based on these points, the technique used by Ficarra $e t$ al. in this study represents a potential advantage. The technique had been used previously $(15,16)$, but these reports were retrospective and had no control groups.

By transposing the ileal conduit from the right to the left body side through a retrosigmoid tunnel a limited mobilisation of the left ureter is sufficient for the anastomosis and there is no angulation of the ureter. A slightly longer ileum segment $(20 v s .15 \mathrm{~cm})$ is needed and a longer and more oblique incision is made in the mesentery at the level of the proximal end which the authors claim to be important to accommodate the retrosigmoid passage of the ileal conduit with no tension. This is an important aspect and potential limitation of the technique which may be problematic in patients with a short mesentery. Since the median body mass index in this series was $26 \mathrm{~kg} / \mathrm{m}^{2}$, it seems that the authors were not able to evaluate whether this technique is also feasible in obese patients, which usually have thicker mesentery that might represent a challenge for the transposition of the ileal segment to the left side. Higher rates of ureteroenteric anastomosis strictures have been reported in patients with high body mass index which seem to require more extensive dissection of the distal ureter (17). In fact, the retrosigmoid ileal conduit might be beneficial for these patients.

The authors claim that their technique might be better than the one involving the transposition of an ileal conduit anteriorly to the sigmoid (18), for keeping the ureteroileal anastomoses completely retroperitoneal. However, it is not possible to make a comparison between the techniques since only this series with the anterior transposition of an ileal conduit has been reported, with similar results. Table 1 compares the results of different techniques (traditional ileal conduit, retrosigmoid ileal conduit and anterior ileal conduit) in terms of ureteroileal strictures.

\section{Limitations of the study}

The study design is nonrandomized, using a contemporary historical cohort as a control group and the sample size is relatively small. In addition, follow-up was short and the control group was followed-up for longer time (27.5 vs. 10.8 months), which may have an impact in the stricture rates considering that the average postoperative time to diagnose UEA strictures can be of 6-18 months (4-6).

Furthermore, the ureteral implantation technique was different in the two groups, with patients in the control group receiving a Wallace ileal conduit, while those in the study group received a Nesbit anastomosis. It remains debatable whether the different techniques of ureteral anastomosis have similar rates of ureteroileal stricture. A recent systematic review with meta-analysis found no differences in this regard between the two techniques (22).

\section{Conclusions}

The retrosigmoid ileal conduit diversion seems to be 
associated with a lower risk of ureteroileal stricture. Further, prospective, randomised studies with larger sample size and long-term follow-up are needed to confirm the promising early results of this technique.

\section{Acknowledgements}

None.

\section{Footnote}

Conflicts of Interest: CM Gomes is a speaker for Astellas, Boston Scientific and Medtronic and is also a member of the advisory board for Boston Scientific. The other author has no conflicts of interest to declare.

\section{References}

1. Lawrentschuk N, Colombo R, Hakenberg OW, et al. Prevention and management of complications following radical cystectomy for bladder cancer. Eur Urol 2010;57:983-1001.

2. Ficarra V, Giannarini G, Crestani A, et al. Retrosigmoid Versus Traditional Ileal Conduit for Urinary Diversion After Radical Cystectomy. Eur Urol 2018. [Epub ahead of print].

3. Madersbacher S, Schmidt J, Eberle JM, et al. Long term outcome of ileal conduit diversion. J Urol 2003;169:985-90.

4. Shah SH, Movassaghi K, Skinner D, et al. Ureteronenteric strictures after open radical cistectomy diversion: the university of Southern Califórnia experience. Urology 2015;86:87-91.

5. Schöndorf D, MeierhansRuf S, Kiss B, et al. Ureteroileal strictures after urinary diversion with an ileal segment is there a place for endourological treatment at all? J Urol 2013;190:585-90.

6. Anderson CB, Morgan TM, Kappa S, et al. Ureteroenteric anastomotic strictures after radical cystectomy - does operative approach matter? J Urol 2013;189:541-7.

7. Tal R, Sivan B, Kedar D, et al. Management of benign ureteral strictures following radical cistectomy and urinary diversion for bladder cancer. J Urol 2007;178:538-42.

8. Richards KA, Steinberg GD. Perioperative outcomes in radicalcystectomy: how to reduce morbidity? Curr Opin Urol 2013;23:456-65.

9. Kramolowsky EV, Clayman RV, Weyman PJ. Management of ureterointestinal anastomotic strictures: comparison of open surgical and endourological repair. J Urol 1988;139:1195-8.

10. Laven BA, O'Connor RC, Gerber GS, et al. Long-term results of endoureterotomy and open surgical revision for the management of ureteroenteric strictures after urinary diversion. J Urol 2003;170:1226-30.

11. DiMarco DS, LeRoy AJ, Thieling S, et al. Long-term results of treatment for ureteroenteric strictures. Urology 2001;58:909-13.

12. Fransen van de Putte EE, de Wall LL, van Werkhoven E, et al. Endo-urological techniques for benign uretero-ileal strictures have poor efficacy and affect renal function. Urol Int 2018;100:18-24.

13. Nassar OA, Alsafa ME. Experience with ureteroenteric strictures after radical cystectomy and diversion: open surgical revision. Urology 2011;78:459-65.

14. Lopes RI, Torricelli FC, Gomes CM, et al. Endovascular repair of a nearly fatal iliac artery injury after endoureterotomy. Scand J Urol 2013;47:437-9.

15. Kotb AF. Ileal conduit post radical cystectomy: modifications of the technique. Ecancermedicalscience 2013;7:301.

16. Li Y, Zhuang Q, Hu Z, et al. A modified ureteroileal anastomosis technique for Bricker urinary diversion. Urology 2011;78:1191-5.

17. Kouba E, Sands M, Lentz A, et al. A comparison of the Bricker versus Wallace ureteroileal anastomosis in patients undergoing urinary diversion for bladder cancer. J Urol2007;178:945-8; discussion 948-9.

18. Pagano S, Ruggeri $P$, Rovellini $P$, et al. The anterior ileal conduit: results of 100 consecutive cases. J Urol 2005;174:959-62; discussion 962.

19. Evangelidis A, Lee EK, Karellas ME, Evaluation of ureterointestinal anastomosis: Wallace vs Bricker. J Urol. 2006;175:1755-8; discussion 1758.

20. Liu L, Chen M, Li Y, Technique selection of bricker or wallace ureteroileal anastomosis in ileal conduit urinary diversion: a strategy based on patient characteristics. Ann Surg Oncol 2014;21:2808-12.

21. Desai MM, Gill IS, de Castro Abreu AL, Robotic intracorporeal orthotopic neobladder during radical cystectomy in 132 patients. J Urol 2014;192:1734-40.

22. Davis NF, Burke JP, McDermott T, et al. Bricker versus Wallace anastomosis: a meta- analysis of ureteroenteric stricture rates after ileal conduit urinary diversion. Can Urol Assoc J 2015;9:E284-90.

Cite this article as: Gomes CM, Laferreira LS. A retrosigmoid ileal conduit might prevent ureteroileal anastomotic stricture after ileal conduit diversion. Transl Androl Urol 2018;7(Suppl 6):S712-S714. doi: 10.21037/tau.2018.10.10 